\title{
Echocardiography of Marfan's Syndrome Patient with New Gene Mutation of FBN1 with 13-year Follow-up
}

\author{
Jianping Xu, MS ${ }^{a, 1}$, Faping Cui, MS ${ }^{b, 1}$, Shuixiu Dou, MS ${ }^{c}$, Jiafu Ou, MD ${ }^{d, *}$ \\ ${ }^{a}$ Department of Ultrasound, The First Hospital of Shanxi Medical University, Taiyuan, Shanxi, China; ${ }^{b}$ Department of Radiology, The \\ 985 Hospital of PLA, Taiyuan, Shanxi, China; ${ }^{\circ}$ Ultrasonic Diagnostic Center, Shaanxi Provincial People's Hospital, Xian, Shanxi, China; \\ ${ }^{d}$ Cardiology Division, Department of Internal Medicine, Washington University in St. Louis, St. Louis, MO, USA \\ Received November 26, 2020; revision received April 10, 2021; accepted April 19, 2021
}

\begin{abstract}
A case of Marfan syndrome was followed up by echocardiography for 13 years to observe the evolution of cardiovascular disease. The initial cardiovascular manifestations of this patient were "mitral myxoid degeneration, chordal rupture, leaflet prolapse, and massive regurgitation". Subsequently, after several years of development, the aortic sinus and ascending aortic aneurysm dilatation appeared and a new gene mutation site G4331A of FBN1 was found by genetic testing in this patient. Whether the new gene mutation site is related to the initial manifestation of the patient's cardiovascular disease with "mitral valve disease" remains to be further verified.
\end{abstract}

Key words: Echocardiography; Marfan syndrome; FBN1 genes; New mutation

Advanced Ultrasound in Diagnosis and Therapy 2021; 03: 249-253

DOI: 10.37015/AUDT.2021.200070

$\mathrm{M}$ arfan syndrome is a connective tissue disease. The incidence is about 2-3/10000. Marfan syndrome is caused by the mutation of FBN1(fibrillin-1 gene) on chromosome 15. At present, more than 1800 mutations of Marfan syndrome have been reported in the human gene mutation database and the UMD-FBN1 mutation database. Marfan syndrome can involve multiple organ systems. The cardiovascular system is characterized by arterial lesions, and aortic dissection; and rupture are the main causes of morbidity and death in patients. We report a case of Marfan syndrome with a new gene mutation site G4331A of FBN1 evaluated by echocardiography with 13 years followed-up to observe the evolution of cardiovascular disease.

\section{Case Study}

A 18-year-old male patient was admitted to the ophthalmology department of the First Hospital of
Shanxi Medical University in June 2004 for poor vision. He was found to have lens subluxation and was recommended to have echocardiography due to his special body shape ( $191.5 \mathrm{~cm}$ height, spider-like fingers). The test results were as follows: posterior mitral valve prolapsed, massive mitral regurgitation, left atrial and left ventricle enlarged (Fig. 1). The patient was clinically suspected to have Marfan syndrome.

The patient had been experiencing chest tightness and excessive sweating since 2000. Those symptoms could usually get better by themselves at first, but the patient continued to have chest tightness and the condition became more and more unstable. The patient was then admitted to a hospital in Beijing in October 2004 with diagnosis of Marfan syndrome and mitral valve inefficacy and was recommended to receive "mitral valvuloplasty". During the operation, extracorporeal circulation was established in the usual fashion and

\footnotetext{
${ }^{1}$ Co-first authors

* Corresponding author: Cardiology Division, Department of Internal Medicine, Washington University in St. Louis, St. Louis, MO, USA. e-mail: jou@wustl.edu
} unrestricted use, distribution and reproduction in any medium provided that the original work is properly attributed. 
incisions were made on the atrium dextrum and interatrial septum to expose mitral valves. The posterior mitral valve was found to be prolapsed, the chordae tendineae were ruptured, and the annulus was obviously

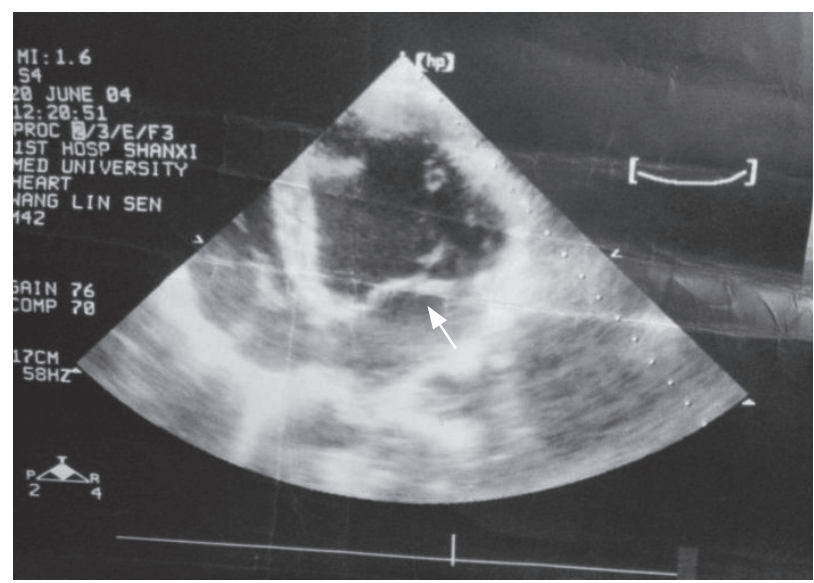

Figure 1 The posterior mitral valve prolapsed as showed by the arrow in the figure.

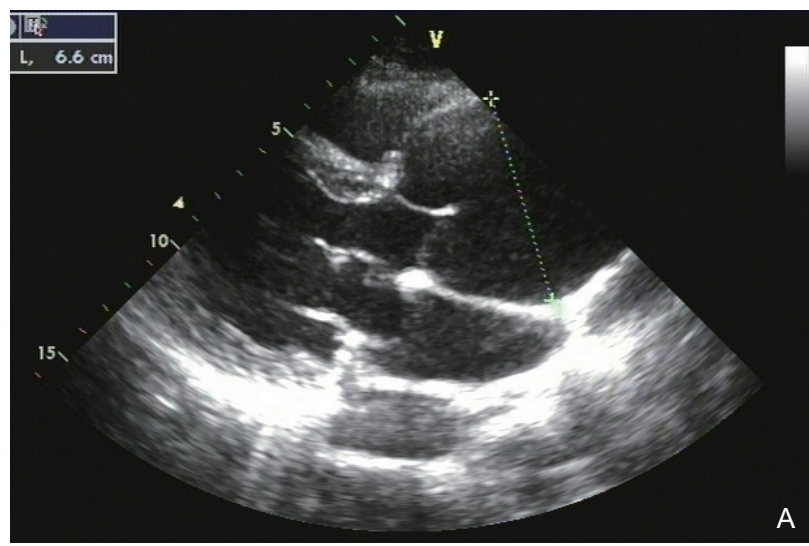

dilated. The posterior lobe without chordae tendineae attached was then surgically removed, the valve ring and the flap section were sutured, and an artificial mitral ring was implanted. One piece of mitral valve tissue (about $3 \times 1 \times 0.25 \mathrm{~cm}$ in size, milky white and soft) was taken for pathological biopsy and the results showed that the patient had myxoid degeneration. Echocardiography was routinely performed in October 2004, April 2005, September 2006, March 2007, February 2009, August 2009, and January 2011, and results are shown in Table 1. Between 2004 and 2007, the patient's mitral valve opened and closed well. In 2009, the aortic sinus of the patient began to expand obviously, and by January 2011, the aortic sinus and proximal ascending aorta were dilated along with a little aortic regurgitation .

In February 2015, the patient underwent cardiac ultrasound in the First Hospital of Shanxi Medical University. It revealed that his aortic sinus and the initial segment of ascending aorta were dilated along with massive aortic regurgitation (Fig. 2).

Figure 2 Ultrasound images of the patient from February 2015 (A)Parasternal Long-Axis View showed that the aortic sinus was dilated up to 66 mm. (B) There was a lot of regurgitation in the aortic valve.

In March 2015, the patient was admitted to a hospital in Beijing with sudden chest and back pain. The chest CTA showed an aortic root tumor whereas abdominal CTA showed no abnormal phenomenon. On April 1, the patient underwent surgical treatment. Dissociation was performed on the lower bottom of the sternum, the right atrium, the ascending aorta, and the aright superior pulmonary vein. Extracorporeal circulation was established in the usual fashion.; After cardiac arrest, the patient was revealed to have thin aortic lobe and aortic valve insufficiency and the aortic sinus and proximal ascending aorta were founded to be dilated with a diameter of about $6 \mathrm{~cm}$. The aortic root was removed and replaced with an artificial tube and the aortic valve was removed and replaced with a mechanical valve. The artificial vessel was directly anastomosed with the coronary artery opening and the distal end of the artificial vessel was anastomosed with the distal end of the ascending aorta. The patient recovered well after surgery.

The patient did genetic testing on September, 2015. The results are shown in Fig. 3. Multiple sequencing alignment of human FBN1 protein with various species revealed that the novel mutation occurred within a highly conserved region of the EGF-like 21Calcium-binding domain (Fig. 4). The patient's parents were alive and well with no abnormalities found in routine physical examination, imaging examination and genetic testing.

\section{Discussion}

Marfan syndrome is a connective tissue disease with autosomal dominant with a incidence of about 2-3/10000 [1]. Typical characteristics of Marfan syndrome include 
abnormally long and slender limbs, fingers and toes, being tall, and heart defects. Cardiovascular damage is very common in patients with Marfan syndrome and is the major cause of their death. The main characteristics of cardiovascular disease are: (1) aortic disease: it is a characteristic change, mainly manifested by main artery dilation, aortic aneurysm, dissecting aneurysm, and aortic annulus - aortic dilation, etc. (2) Aortic insufficiency; (3) mitral valve prolapse; (4) biphasic murmurs can be heard at apex of heart [2]. The disease may also affect other organs, such as lung, eye, dura, hard jaw, etc $[3,4]$.

\begin{tabular}{|c|c|c|c|c|c|c|c|c|c|}
\hline \multicolumn{2}{|c|}{ Clinical diagnosis } & \multicolumn{8}{|c|}{ Suspected Marfan syndrome } \\
\hline \multicolumn{10}{|c|}{ Testing information } \\
\hline \multicolumn{2}{|c|}{ Test content } & \multicolumn{8}{|c|}{ Genetic testing for hereditary aortic disease } \\
\hline \multirow{3}{*}{\multicolumn{2}{|c|}{ Gene tested }} & \multicolumn{8}{|c|}{15 genes associated with hereditary aortic aneurysms } \\
\hline & & FBN1 & TGFB2 & SMAD3 & TGFBR1 & TGFBR2 & ACTA2 & MYH11 & SMAD4 \\
\hline & & MYLK & NOTCH1 & PRKG1 & SKI & COL3A1 & SLC2A10 & FBN2 & \\
\hline \multicolumn{3}{|c|}{ Detection method } & \multicolumn{7}{|c|}{ Gene combination testing } \\
\hline \multicolumn{10}{|c|}{ Testing results } \\
\hline Gene & \multicolumn{2}{|c|}{ The reference sequence } & \multicolumn{2}{|c|}{ Genetic way } & Mutation site & Amino acid changed & \multicolumn{2}{|c|}{ Homozygous or heterozygous } & Pathogenicity \\
\hline FBN1 & \multicolumn{2}{|c|}{ NM-000138 } & \multicolumn{2}{|c|}{ Autosomal dominant inheritance } & c. $4331 \mathrm{G}>\mathrm{A}$ & p.Cys1444Tyr & \multicolumn{2}{|c|}{ heterozygous } & May be harmful \\
\hline
\end{tabular}

Figure 3 Gene Testing Report of Aortic Disease

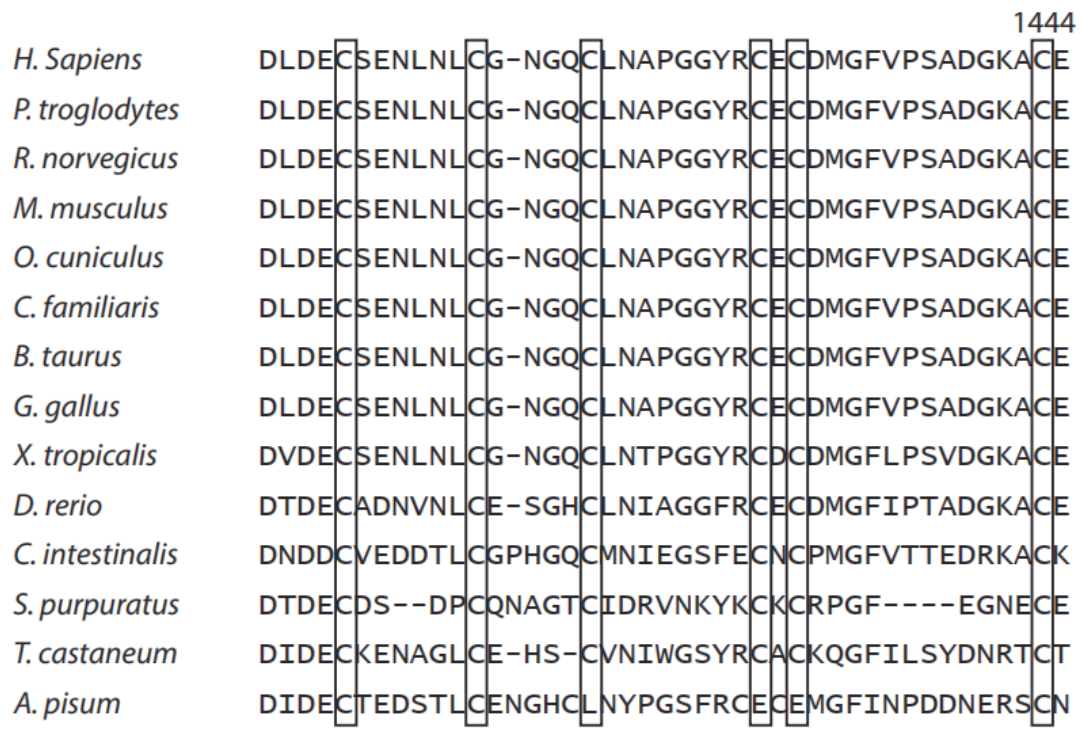

Figure 4 Orthologous protein sequence alignment of FBN1 from different species, the mutated residue showing conservation of cysteine at codon 1444.

Marfan syndrome is caused by the mutation of FBN1(fibrillin-1 gene) on chromosome 15. FBN1 was identified and cloned by Dietz [5] in 1991 using in situ hybridization. The gene was located at $15 \mathrm{q} 2.1$ cDNA, with a full length of $200 \mathrm{~kb}$, containing 65 exons, a coding sequence of $10 \mathrm{~kb}$, and a mRNA of 9663 nucleotides. The open reading frame was 8613 nucleotides. Fibrinogen 1 with a cysteine-rich $320 \mathrm{kDa}$ glycoprotein, is the product of FBN1[6,7]. The precursor of fibrinogen 1 contains several specially structured regions, such as the epidermoid growth factor (EGFlike) and transforming growth factor binding protein (LTBP). Cellulose is one of the main components of microfibrils or elastic fibers. It is widely distributed in aorta, suspensory ligament of lens and periosteum. The hyperextension of connective tissue, caused by abnormal fibrinogen, makes patients more likely to have premature severe cystic meiosis, which usually leads to autoctisis and phacomatosis. Bone defects can be the indirect results of the hyperextension of connective 
tissue as it covers the surface of the periosteum and provides a counterforce during the normal growth of connective tissue. As the elasticity of the periosteum increases, bone overgrowth occurs.

Table 1 Echocardiography results over the years from 2004 to 2011

\begin{tabular}{|c|c|c|c|c|c|c|}
\hline Data & $\begin{array}{c}\text { The diameter of } \\
\text { aortic sinuses }(\mathrm{mm})\end{array}$ & $\begin{array}{l}\text { The diameter of } \\
\text { aortic sinotubular } \\
\text { junction }(\mathrm{mm})\end{array}$ & $\begin{array}{l}\text { The diameter of } \\
\text { ascending aorta } \\
(\mathrm{mm})\end{array}$ & $\begin{array}{c}\text { The anteroposterior } \\
\text { diameter of the left } \\
\text { atrium }(\mathrm{mm})\end{array}$ & $\begin{array}{l}\text { The anteroposterior } \\
\text { diameter of left } \\
\text { ventricular }(\mathrm{mm})\end{array}$ & $\begin{array}{l}\text { Left ventricular } \\
\text { ejection fraction(\%) }\end{array}$ \\
\hline October 2004 & 38 & 31 & 30 & 25 & 51 & 51 \\
\hline April 2005 & 38 & 30 & 30 & 28 & 55 & 55 \\
\hline September 2006 & 39 & 30 & 30 & 28 & 50 & 50 \\
\hline March 2007 & 39.6 & 31 & 30 & 28 & - & - \\
\hline February 2009 & 43 & 33 & 30 & 22 & 52 & 52 \\
\hline August 2009 & 46 & 35 & 31 & 22 & 52 & 52 \\
\hline January 2011 & 46 & 36 & 36 & 20 & 53 & 53 \\
\hline
\end{tabular}

At present, more than 1800 mutations of Marfan syndrome have been reported in the human gene mutation database and the UMD-FBN1 mutation database $[8,9]$. The mutation site of this patient was FBN1. G4331A, which made the amino acid 1444 of the encoded protein fibrillin-1 change from cysteine into tyrosine (CYS1444TYR). To our knowledge, this mutation site was not reported in the previous databases and published papers. Genetic tests of the patient's parents showed no abnormalities. The mutation site FBN1G4331A is in Exon 34 (4211-4336), which is within the EGF-like 21Calcium-binding region and just in front of the CBEGF22-TB4-CBEGF23 (PDB 1UZJ) region. Cys 1444 is highly conserved in evolution. The epidermoid growth factor structural regions of FBN1 play a major role in the pathogenesis of fibrinosis. Hence, most mutations in these structural regions are associated with classical MFS [10]. Each epidermoid growth factor structural region contains six highly conserved cysteine residues, which form the consensus sequence $\mathrm{NH} 2$ terminal region binding disulfide bonds (C1-C3, C2$\mathrm{C} 4, \mathrm{C} 5-\mathrm{C} 6)$ and calcium within the three regions [11]. Since three disulfide bonds are required to maintain normal conformational folding in the EGF-like structural region, the loss or addition of cysteine residues can lead to abnormal conformational folding [12]. Combined with the clinical manifestations and the genetic testing results of this patient, we highly suspect that this new mutation locus G4331A is the pathogenic mutation. The patient's parents have no manifestation of Marfan's syndrome and no abnormalities in genetic testing, which indicates that at least $25 \%$ of cases are new mutations. Usually, aortic lesions are the characteristic lesions of Marfan's syndrome, but "mitral myxoid degeneration, chordal rupture, leaflet prolapse, and massive regurgitation" were the patient's initial presentation. After years of development, the aneurysm of the aortic root appeared. Whether this cardiovascular manifestation is related to this gene mutation locus FBN1, G4331A needs continuing research. Clinically, the initial presentation of this patient with massive mitral regurgitation may be related to early chordae tendinae rupture. The presentation of severe aortic root aneurysm at the age of 29 is consistent with the common presentation of patients with Marfan syndrome.

International diagnostic criteria for Marfan syndrome were first published in 1988 [13]. With the discovery that FBN1 gene mutation leads to Marfan's syndrome, Ghent-1 diagnostic criteria were proposed in 1991 and the pathogenic FBN1 gene mutation was taken as one of the main criteria for diagnosis [14]. To simplify and specify the diagnostic procedure of Marfan's syndrome, the diagnostic criteria for Ghent-1 were updated in 2010 [15]. As specificity increases, sensitivity decreases. Although he patient was not tested for FBN1 gene mutation before the first mitral valve operation, which did not meet the diagnostic criteria of Marfan syndrome, it was clinically highly suspected that he had Marfan syndrome. The patient continued to recheck the cardiac ultrasound after the surgery. From 2011 to 2015, patients failed to follow up, fortunately, nothing happened. The patient was diagnosed with Marfan's syndrome at the second surgery. After the surgery, the patient was tested for a mutation in the FBN1 gene and a new mutation that had not been reported was found. It even occurred in highly conserved cysteine. Therefore, we think it is highly likely that this is the mutation that causes the disease, though further investigation is needed. 


\section{Conclusion}

In this case, we found a new gene mutation locus G4331A of FBN1, which enriches the genotype spectrum and can provide a basis for accurate diagnosis and genetic counseling of this disease. The mutation of FBN1 gene in this patient occurred in highly conserved cysteine, which we believe is highly likely to be the pathogenic mutation, though further confirmation is needed.

\section{Acknowledgments}

We thank the patient who participated in this study. We are grateful to all the staff involved in this study.

\section{Conflict of Interest}

The authors have declared that no comflict interests exist.

\section{Reference}

[1] Gritti A, Pisano S, Catone G, Iuliano R, Salvati T, Gritti P. Psychiatric and neuropsychological issues in Marfan syndrome: A critical review of the literature. Int J Psychiatry Med 2015; 50: 347-360.

[2] Shuqin L, Dingyin Z. Cardiovascular changes in 26 cases of Marfan's syndrome. Chinese Journal of Practical Internal Medicine 1992, 12: 78-79.

[3] Reyes-Hernández OD, Palacios-Reyes C, Chávez-Ocaña S, CortésMalagón EM, Alonso-Themann PG, Ramos-Cano V, et al. Skeletal manifestations of Marfan syndrome associated to heterozygous R2726W FBN1 variant: sibling case report and literature review. BMC Musculoskelet Disord 2016; 17: 79.

[4] Aubart M, Gross MS, Hanna N, Zabot MT, Sznajder M, Detaint D. The clinical presentation of Marfan syndrome is modulated by expression of wild-type FBN1 allele. Hum Mol Genet 2015; 24: 2764-2770.
[5] Dietz HC, Cutting GR, Pyeritz RE, Maslen CL, Sakai LY, Corson GM, et al. Marfan syndrome caused by a recurrent de novo missense mutation in the fibrillin gene. Nature 1991; 352: 337-339.

[6] Wenjiao X, Xue G, Hong G. Screening for pathogenic genes in a family of Marfan syndrome. International Eye Science 2016, 16: 948-951.

[7] Ying B, Ning L, Quancheng K, Jun Y, Qinghua W, Zhenhua Z, et al Analysis of FBN1 pathogenic gene mutations in a family of Marfan syndrome and prenatal diagnosis. National Medical Journal of China 2015, 95: 145-146.

[8] Faivre L, Collod-Beroud G, Callewaert B, Child A, Loeys BL, Binquet C, et al. Pathogenic FBN1 mutations in 146 adults not meeting clinical diagnostic criteria for Marfan syndrome: further delineation of type 1 fibrillinopathies and focus on patients with an isolated major criterion. Am J Med Genet A 2009 ; 149: 854-860.

[9] Xiangqun J, Zhi X, Liqiong W. Clinical features of Marfan syndrome and analysis of FBN1 gene mutation. Journal of Clinical Pediatrics 2016, 34: 775-778.

[10] Schrijver I, Liu W, Brenn T, Furthmayr H, Francke U. Cysteine substitutions in epidermal growth factor-like domains of fibrillin-1: distinct effects on biochemical and clinical phenotypes. Am J Hum Genet 1999; 65: 1007-1020.

[11] Reinhardt DP, Ono RN, Sakai LY. Calcium stabilizes fibrillin-1 against proteolytic degradation. J Biol Chem 1997; 272: 1231-1236.

[12] Downing AK, Knott V, Werner JM, Cardy CM, Campbell ID, Handford PA. Solution structure of a pair of calcium-binding epidermal growth factor-like domains: implications for the Marfan syndrome and other genetic disorders. Cell 1996; 85: 597-605.

[13] Beighton P, de Paepe A, Danks D, Finidori G, Gedde-Dahl T, Goodman R, et al. International nosology of heritable disorders of connective tissue, Berlin, 1986. Am J Med Genet 1988; 29: 581-594.

[14] De Paepe A, Devereux RB, Dietz HC, Hennekam RC, Pyeritz RE. Revised diagnostic criteria for the Marfan syndrome. Am J Med Genet 1996; 62: 417-426.

[15] Loeys BL, Dietz HC, Braverman AC, Callewaert BL, De Backer J, Devereux RB, et al. The revised Ghent nosology for the Marfan syndrome. J Med Genet 2010; 47: 476-485. 